\title{
The Impact of Networking on Image Acquisition System Design
}

\author{
John H. Perry
}

Widespread integration of networking technology will change the hospital imaging environment, making computational, archiving, and film production services available to network-connected modalities. This change will be reflected in the designs of the image acquisition modalities themselves. A view of the future is presented in which the modalities depend on network-provided services, simplifying the modalities' designs and making them more cost-effective. Achieving this view will require cooperative efforts by standards groups, PACS and modality manufacturers, and hospital organizations. Copyright (C) 1991 by W.B. Saunders Company

$\mathbf{B}^{\mathrm{r}}$ Y THE TWENTY-FIRST century, the imaging environment will have changed substantially, driven by many of the technologies currently grouped under the label "picture archiving and communications system" (PACS). Connectivity among image acquisition modalities and service components on networks will precipitate structural changes in modalities themselves, making them generally less complex, less expensive, and more efficient. The integrated imaging environment of the twenty-first century will be viewed as a single system rather than an interconnected collection of independent systems.

The objectives of the integrated imaging environment are (1) better cost-effectiveness at the system level, (2) better diagnostic performance, (3) greater speed in handling the entire medical record, and (4) greater security in managing the entire medical record. To meet these objectives, the integrated imaging environment must supply at least the following services: image acquisition system integration, hospital information system (HIS)/radiology information system (RIS) integration, data base query support for all connected data bases, image and report storage and retrieval, film handling, and viewing. Image and report distribution is important not only for the internal efficiency of the hospital but also for better integration of the referring physician into the imaging process. Image acquisition modalities will

From Siemens Gammasonics, Hoffman Estates, $I L$. Address reprint requests to John Perry, Siemens Gammasonics, 2501 N Barrington Rd, Hoffman Estates, IL 60195.

Copyright (C) 1991 by W.B. Saunders Company

0897-1889/91/0404-0103\$03.00/0 change in response to the availability of such services.

Image acquisition modalities today may be parsed into subsystems in the following categories: patient interface, image data acquisition system, image processing system, operator's control system, physician's viewing system, image archival system, and image documentation system. This model applies to a wide spectrum of modalities, from computed tomography (CT), magnetic resonance imaging (MRI), and nuclear medicine (NM) systems to digital radiography.

In the case of a CT system, for example, the patient interface is the couch; the data acquisition system is the gantry, detector, and front-end electronics; the image processing system is the reconstruction hardware and software; the operator's control system is a console with its associated computer; the physician's viewing system is generally a separate console; the image archival system is usually a magnetic tape or optical disk drive; and the image documentation system is usually a camera connected to either the operator's control system or the physician's viewing system, the latter then becoming primarily a filming station.

With the advent of PACS technology, some of these functions will be more effectively accomplished by network-connected service components; eg, separating the physician's viewing system, the image archival system, and the image documentation system from the modality would simplify the modality while providing the services more generally and consistently in an institution. An additional interesting possibility is use of PACS workstations as operator's control systems, further simplifying the design of the core modality.

To achieve this restructuring, some image acquisition architectures will have to change more than others. Figure 1 shows a simplified diagram of an existing generic CT or MRI system. In this system, the functions of data acquisition, image processing, operator's control, physician's viewing, and archiving are combined in hardware at the lowest level in the system, making the functions not easily separable. 


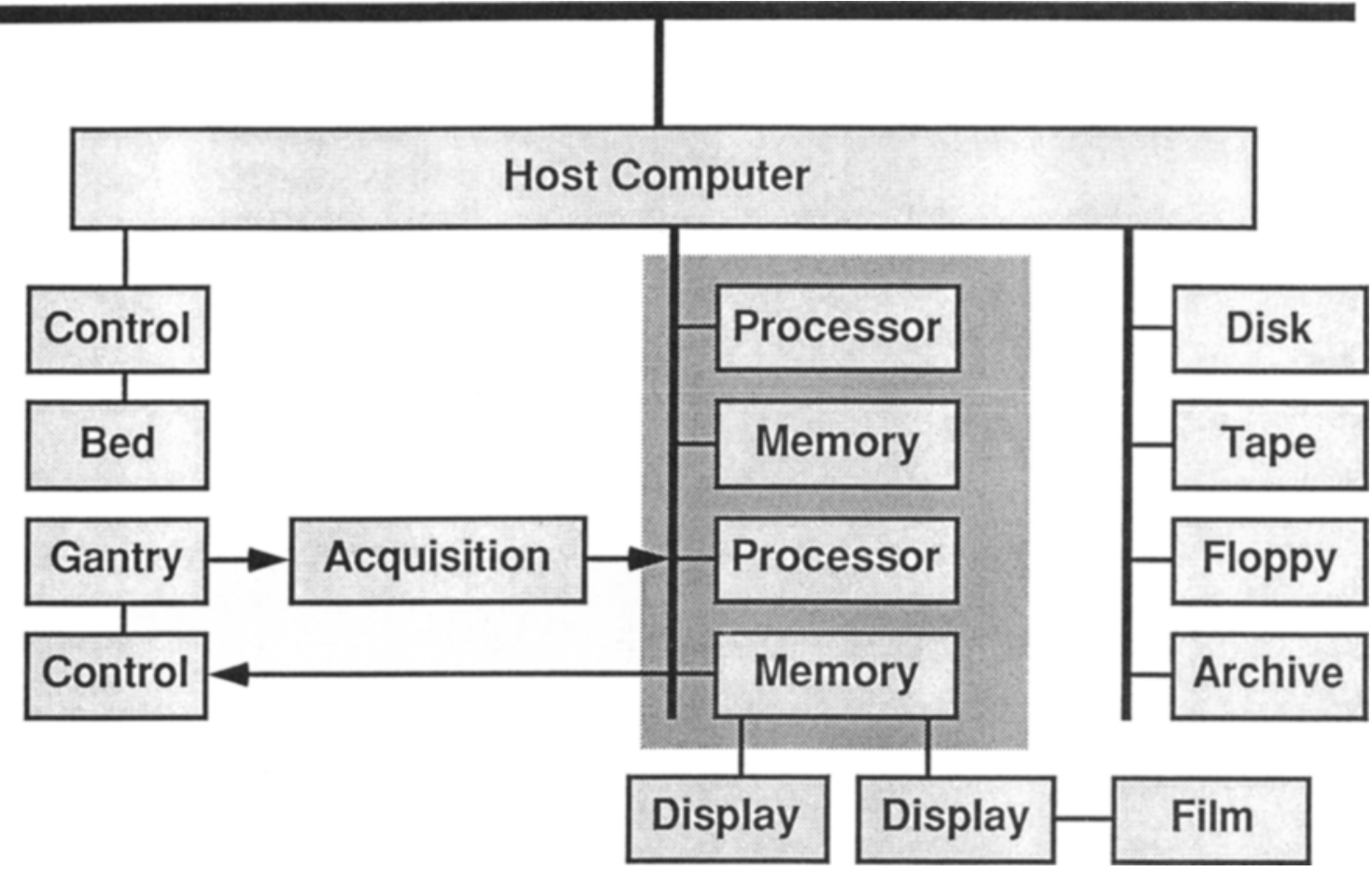

Fig 1. Existing generic CT or MRI system.

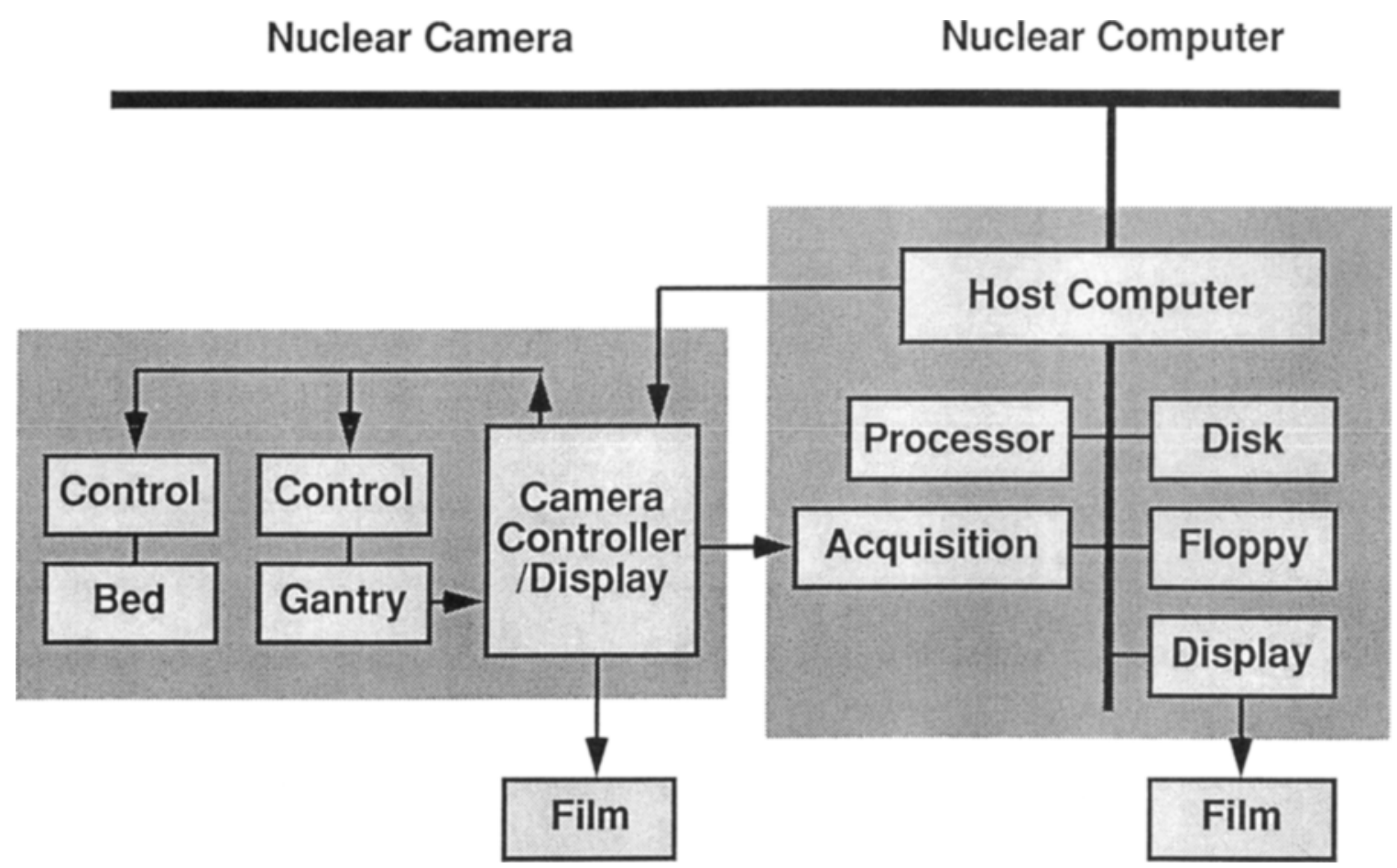

Fig 2. Existing nuclear medicine system. 


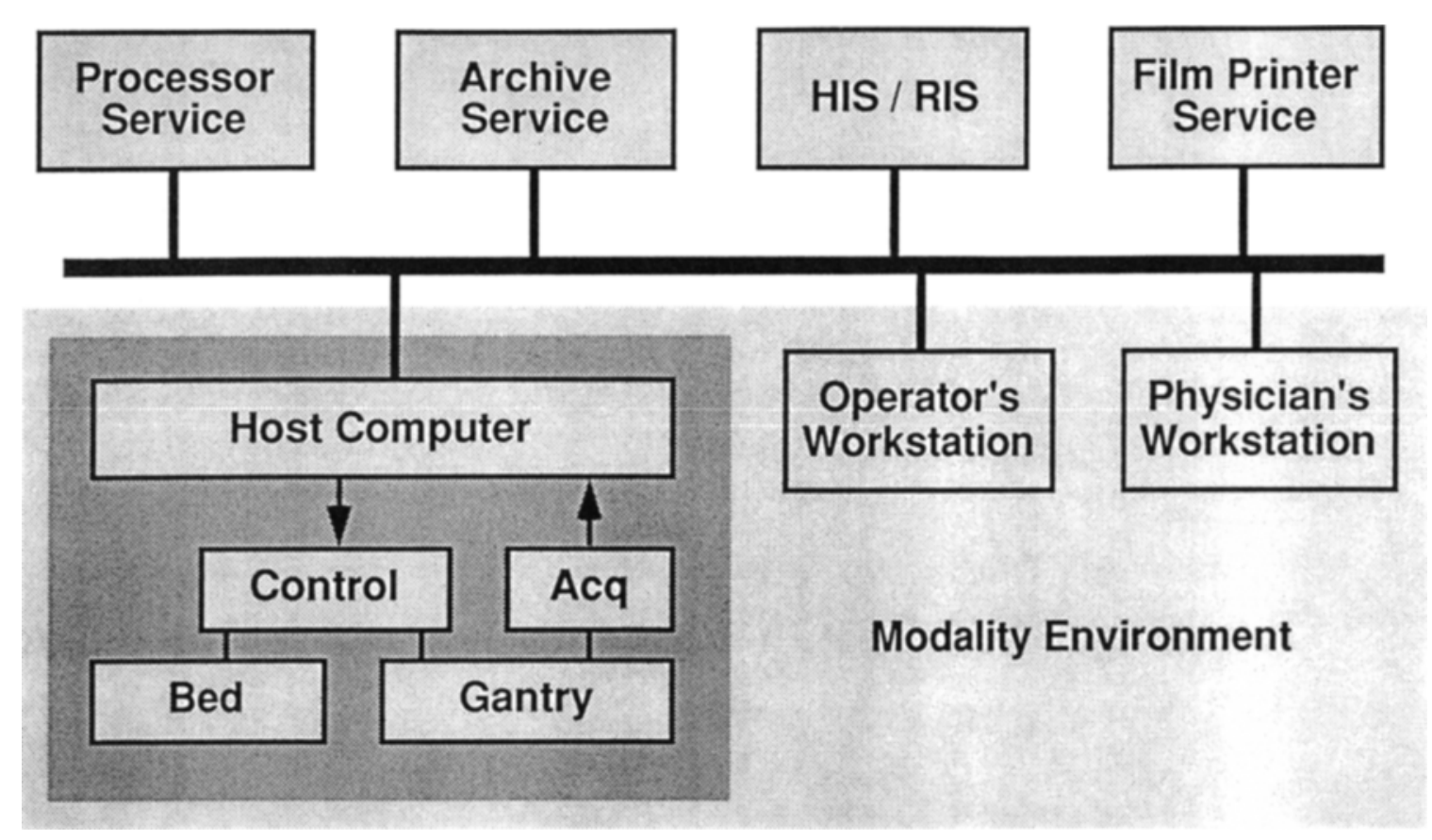

Fig 3. Future generic modality.

The NM system has for many years been structured differently. Gamma camera systems contain a patient couch, gantry, and the detector with its associated electronics. Desktop workstations provide operator's control, acquisition, and image processing functions. Other desktop workstations, networked to the acquisition workstations, provide physician's viewing capability. Archiving is sometimes handled at the workstation level and sometimes at the network level.

Figure 2 shows a diagram of an existing NM system. A unique aspect of $\mathrm{NM}$ is the existence of a de facto standard interface between the camera system and the nuclear computer. This allows separation of the functions more easily than in current CT and MRI systems. NM systems of the very near future will define these interfaces even more clearly.

The vision of the future is that CT and MRI will evolve in this way as well, becoming acquisition engines that receive services from networkconnected components for data base, archival, and film production, as well as operator's and physician's workstations and even specialized processing, as shown in Fig. 3. The resulting global environment will appear as in Fig. 4.
The network-connected workstation will be a major factor in achieving the objective of simplifying image acquisition systems. There will be two forms of workstation: very-high-performance systems dedicated to primary diagnosis, and medium-performance systems for use in operator's control of modalities, physician's image review at modalities, remote image and report review, teleradiology, and transcription. The technical functions of such a workstation fall into two groups: those which are native to the workstation, and those which the workstation can access from other network-connected service components. All workstations will be required to provide a core of viewing functions, including some modalityspecific functions such as measurement, multiplanar reconstruction, and some three-dimensional processing. In addition, workstations will have to access external services including data base services from HIS, RIS, and PACS, as well as processing services for operator's control programs and specialized image processing. Interfaces between PACS and HIS and RIS will affect the structure of the operator interfaces of modalities through automatic acquisition of the patient's demographic information as well as 


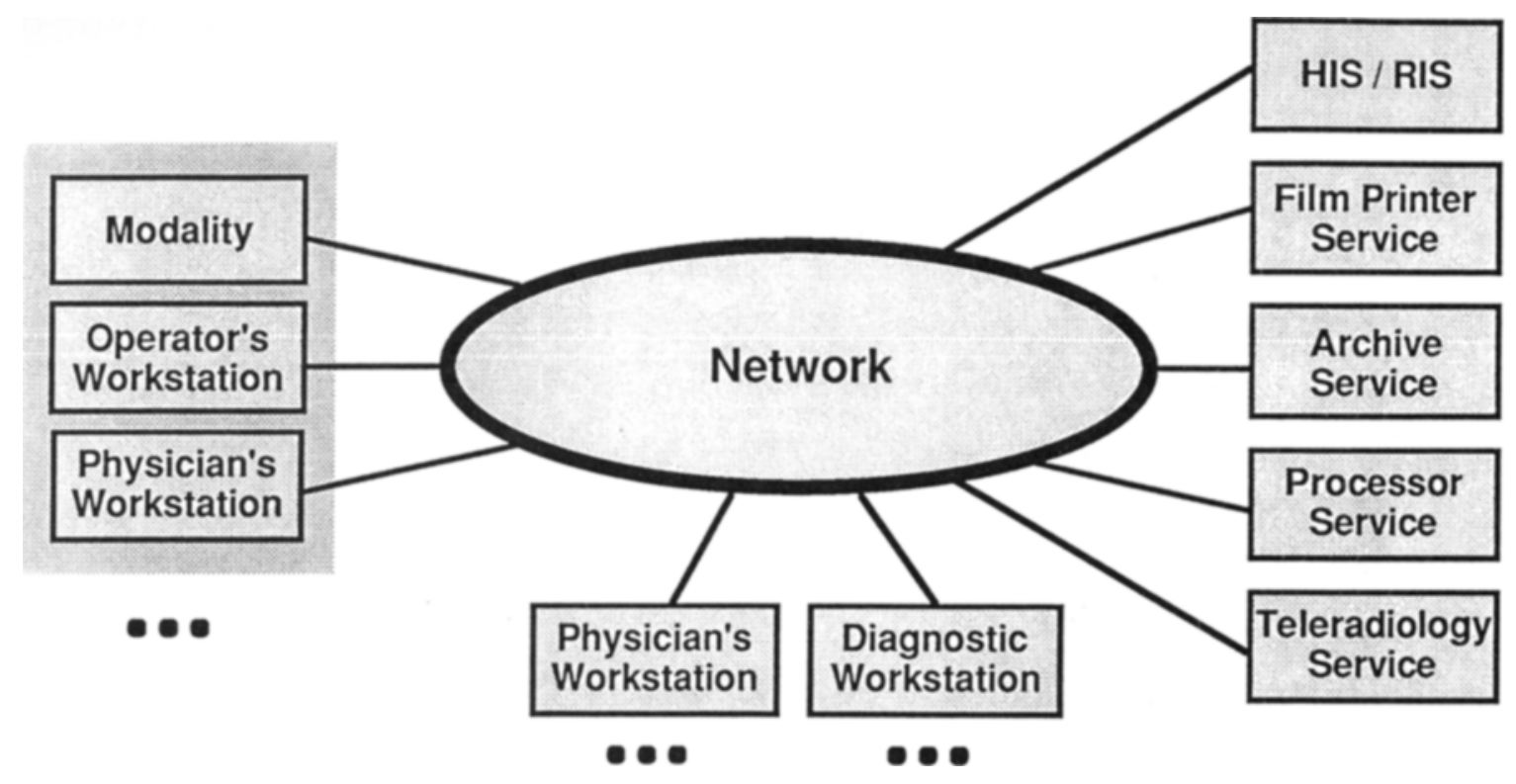

Fig 4. Integrated imaging environment.

through provision of worklists to modality operators.

To achieve all these goals in a multivendor imaging environment, standards are necessary. The ACR/NEMA standard has been a useful step in this direction; however, for it to play a major role in the future of the imaging environment, it must be expanded to accommodate HIS/RIS connectivity, off-line storage media formats, and true networking. In addition, however, presentation-layer protocols such as $\mathrm{X}$ windows must be accepted as ways to provide access to external processing services for operator's control programs.

In addition, other organizational issues must be resolved throughout the medical imaging community. Among those issues are:

1. Manufacturers' internal organizations must integrate their activities. PACS and modality engineering and marketing groups must work together to incorporate standards for communication and storage into all their products.

2. Manufacturers must cooperate among themselves to agree on standards. In this regard, ACR/NEMA can only be viewed as a beginning.

3. Hospitals' internal organizations must cooperate to assimilate the technology. In some cases, this will require redefinition of the responsibilities for image management; eg, in some cases computer systems departments may be better positioned to operate the electronic file room than the radiology department. Trust and cooperation will be necessary to achieve the goals.

4. Finally, hospitals themselves must be prepared to support technical solutions to the problems of the digital imaging age. 